\title{
Superficial fungal infections: clinical and epidemiological study in adolescents from marginal districts of Lima and Callao, Peru
}

\author{
Juan Medina Flores ${ }^{1}$, Vilma Bejar Castillo², Florencio Cortez Franco ${ }^{3}$, Armando Betanzos \\ Huata $^{3}$ \\ ${ }^{1}$ Children's Health National Institute, Department of Pediatrics \\ ${ }^{2}$ Mycology section of the Tropical Medicine Institute, Daniel Alcides Carrion National Hospital, Callao, Peru \\ ${ }^{3}$ Department of Dermatology, Daniel Alcides Carrion National Hospital, Callao, Peru
}

\begin{abstract}
Background: Since limited data on superficial fungal infections in teenagers exist in our setting, this study provides the first description of the clinical and epidemiological characteristics of these infections among teenagers in Lima and Callao, Peru. Methodology: The study involved 1,387 adolescents in five public schools from June to November 2006. Participants were examined for superficial fungal lesions. Samples of skin scrapings for microbiological investigations were obtained from suspicious lesions.

Results: A total of 257 subjects were identified with suspected superficial fungal infections. Microbiological assessment was positive for 166 of 257 (64.59\%). The average prevalence was $12.61 \%$ with variation between different districts. Males were more affected $(64 \%)$ than females $(36 \%)(\mathrm{p}=0.001)$. Pet ownership, use of public baths, and wearing sneakers were identified as important risk factors. The majority $(61.5 \%)$ of the subjects presented with itching although $38.5 \%$ were asymptomatic. Tinea pedis was observed in $62.6 \%$, onychomycosis in $24 \%$ and pityriasis versicolor in $10.8 \%$. Dermatophytes were isolated in 105 cases with T. rubrum being identified in 86 cases (59.7\%), T. mentagrophytes in $14(9.7 \%)$ and yeast in 39 (23.4\%). Malassezia spp. was found by direct examination in 18 cases (12.5\%), C. kruseii in 8 cases (5.6\%), and C. albicans in 2 cases (1.4\%). Mixed infections were found in 22 cases.

Conclusions: Superficial fungal infection manifesting as tinea pedis, onychomycosysis and tinea versicolor is prevalent in our setting. As many infections remain asymptomatic, regular examination of this population is advocated. The associated risk factors for these infections also need to be addressed.
\end{abstract}

Keywords: Adolescent, fungal, infection, superficial

J Infect Dev Ctries 2009; 3(4):313-317.

Received 10 August 2008 - Accepted 16 December 2008

Copyright $\odot 2009$ Flores et al. This is an open-access article distributed under the Creative Commons Attribution License, which permits unrestricted use, distribution, and reproduction in any medium, provided the original work is properly cited.

\section{Introduction}

Fungal skin infections are categorized into superficial and deep infections. Superficial infections are limited to the epidermis, hair, nails and the mucous membrane; the three most common types are dermatophytosis, tinea versicolor and candidiasis, while the least common are tinea negra tinea piedra, and the infections caused by nondermatophytic moulds. It is known that the prevalence of these infections, like the ones caused by dermatophytosis, tend to change with age, geographic location, and climate $[1,2]$. Hence, in some regions, zoophilic dermatophytes are isolated more frequently in children and adolescents with tinea capitis, tinea corporis and tinea faciae [3].

The World Health Organization (WHO) defines adolescence as the stage between 10 and 19 years of age; however, the National Institute of Statistics and Data processing of Peru classifies adolescents between the ages of 12 and 17 years old, and this group constitutes $11.16 \%$ of the population in the provinces of Lima and Callao [4]. This age group has a high risk of contracting a superficial mycotic infection, due to youths' high participation in sporting activities and attendance at public baths. Tinea capitis is uncommon in this age group, most likely because of the higher content of fungistatic fatty acids in 
the sebum and the acid mantle quality [5]. In contrast, this group may have high frequencies of pityriasis versicolor infection, probably because during puberty the increasing levels of sexual hormones modulate distribution of grease and sebaceous gland secretions, which are the key elements in the metabolism of the lipophilic yeasts of the Malassezia [6]. In addition, onychomycosis, which is a recurrent chronic infection caused by dermatophytes, yeasts and other opportunistic fungus, has been observed in adolescents.

Superficial fungal infection is the most common ailment for teenagers seeking medical attention from dermatologists. The presence of these lesions may negatively affect the development of teenagers' personalities and selfesteem [7]. As there is paucity of data on the occurrence of superficial fungal infection among teenagers in our setting, this study was designed to assess the prevalence, risk factors, and etiological agents of these infections in the marginal districts of Lima and Callao.

\section{Materials and Methods}

The observational and prospective study was conducted between June and November 2006. The research for cases of superficial mycotic infections was performed in adolescents from five schools located in the districts of Ventanilla, El Agustino, Comas, San Juan de Lurigancho, and San Juan de Miraflores. As there is no reported literature on the prevalence of superficial mycotic infections in adolescents for Peru, the sample size was determined by using statistical data of the number of patients in this age group seen at the Dermatology Service of the Specialized Institute of Child Health in Lima. Calculation of sample size was conducted using the epidemiological software, Epi Info 2002. Based on an estimated prevalence of $11.94 \%$, the minimum sample size was determined to be 162 clinical cases. Assuming $60-80 \%$ of the samples could be positive for infection, at least 257 adolescents with suspicious lesions have to be examined, which was calculated with $11.94 \%$ as a parameter of prevalence.

Adolescents between 12 and 17 years old, who attended a national school in marginal districts located in the provinces of Lima and Callao, were eligible for enrolment in the study.
Exclusion criteria included the presence of endocrine and immunologic diseases, as well as the use of systemic and topic corticoids and systemic antimycotics within 30 days prior to examination, and the application of antimycotic creams during the preceding week. Informed consent was obtained from the parents or legal guardians of the students. Students enrolled in the study were interviewed and a clinical evaluation was performed. Demographic data and information on house pets, the use public baths without wearing slippers, wearing running shoes, participating in sports activity and infection history of scalp, skin and nails were collected.

Samples of scrapings from the skin and nails of students with suspicious lesions were obtained for microbiological analysis. The samples were collected in sterile petri plates and sent to the Mycology section of the Tropical Medicine Institute at the National University of San Marcos, where a direct exam with $20 \% \mathrm{KOH}$ was conducted, and then the sample was cultivated in Sabouraud agar. Micro-cultivation of positive cultures was performed for the identification of dermatophytes. For candida, a test of the germ tubes and carbohydrate fermentation was performed.

Statistical analysis was carried out using Statistical Package for the Social Sciences (SPSS) v. 13.0.

\section{Results}

We examined 1,361 adolescents and 257 were clinically diagnosed with a superficial mycotic infection. Laboratory analysis of clinical samples confirmed the diagnosis in 166 of the 257 cases, yielding a prevalence of $12.61 \%$. Variation of infections occurred among students in different districts with the highest prevalence found in Agustino with 18.27\% (38/208), followed by Ventanilla with $13.85 \%$ (32/231), San Juan de Miraflores with 12.68\% (43/339), Comas with $9.31 \%$ (19/204), and San Juan de Lurigancho with $8.97 \%$ (34/379). Males were affected more than females $(64 \%$ vs. $36 \%$ respectively; $\mathrm{p}=0.001$ ). 
Table 1. The identified dermatophytes and yeast.

\section{$\begin{array}{lll}\text { DERMATOPHYTES AND YEAST NUMBER PERENTAGE } & \text { PUT }\end{array}$}

\begin{tabular}{lcc}
\hline T. rubrum & 86 & 59.7 \\
\hline T. mentagrophytes & 14 & 9.7 \\
\hline T. tonsurans & 4 & 2.8 \\
\hline $\begin{array}{l}\text { T. rubrum } \\
\text { T. mentagrophytes }\end{array}$ & 1 & 0.7 \\
\hline Malassezia spp. & 18 & 12.5 \\
\hline C. kruseii & 8 & 5.6 \\
\hline C. tropicalis & 5 & 3.5 \\
\hline C. glabrata & 4 & 2.8 \\
\hline C. albicans & 2 & 1.4 \\
\hline C. famata & 1 & 0.7 \\
\hline C. guillermondi & 1 & 0.7 \\
\hline TOTAL & 144 & 100
\end{tabular}

Figure 1. The affected corporal regions.

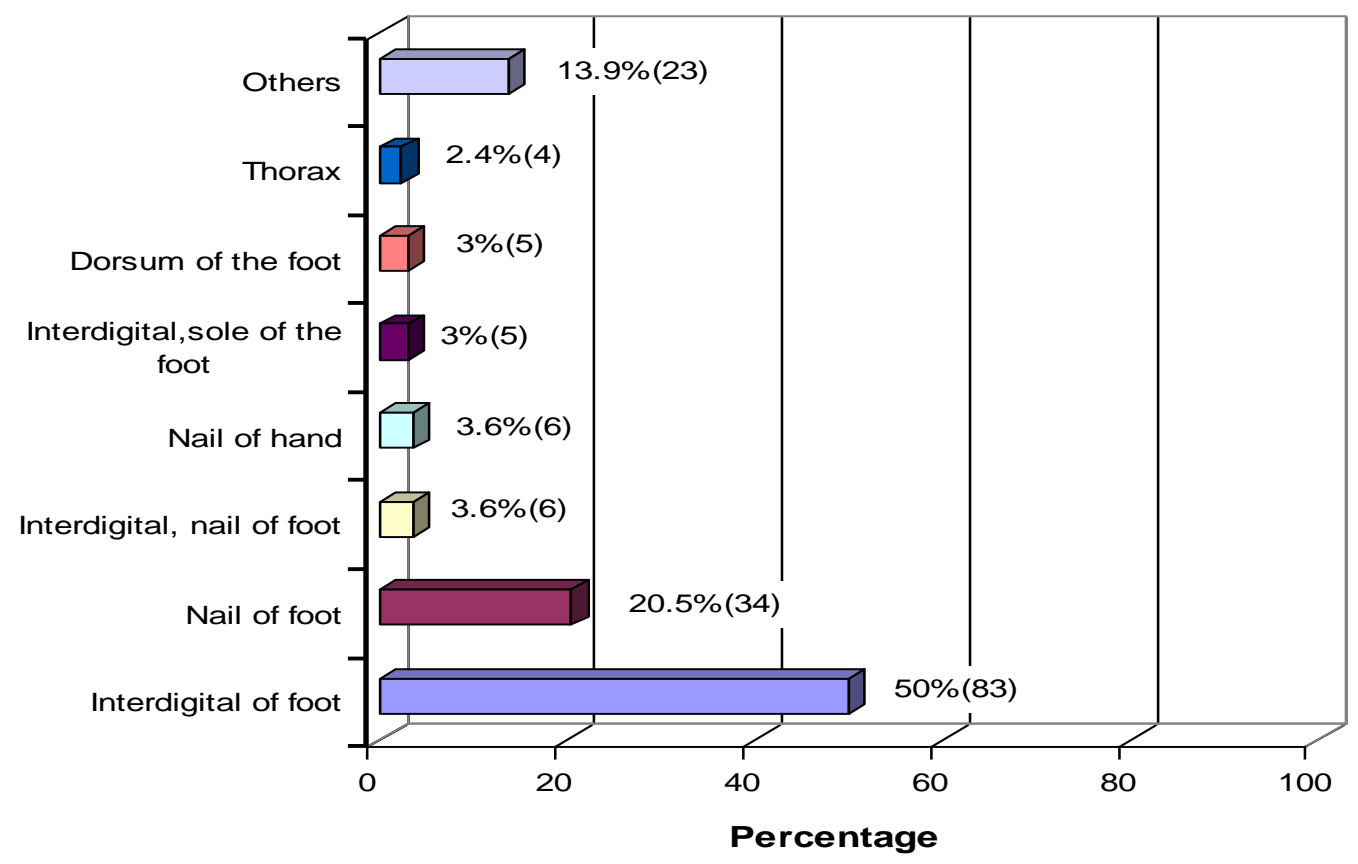


Among positive cases for dermatophytes, $61.2 \%$ had dogs, $38.7 \%$ had cats, and $16.2 \%$ had birds as pets. The use of sneakers and public baths were both reported by $72 \%$ of those with infections, and the majority (62\%) played football. Itching was the sole symptom reported in two-thirds of cases although about $38.5 \%$ were asymptomatic. Clinical examination revealed the presence of exfoliative areas in $71.5 \%$ of the students, while $24.5 \%$ had interdigital fissures, and only $5.9 \%$ had presence of exudates. Of the 166 cases confirmed by cultivation, the most frequent diagnoses were the following: tinea pedis in $62.6 \%$ of cases, onychomycosis in $24 \%$ and pityriasis versicolor in $10.8 \%$. The most commonly affected anatomical region was the interdigital region of the feet $(50 \%)$, followed by the toenails (20.5\%). Figure 1 shows the distribution of the body sites affected. The isolated fungal agents were dermatophytes in 105 cases $(63.3 \%)$, yeasts in 39 cases $(23.4 \%)$, and mixed infections in 22 cases (13.3\%). Table 1 shows the distribution of the identified etiological agent.

\section{Discussion}

Our study showed that the prevalence of the superficial fungal infections in teenagers from marginal districts in the provinces of Lima and Callao was $12.61 \%$. The study also identified the common risk factors for these infections. The prevalence varied from $8.97 \%$ in one district to as high as $18.27 \%$ in another district. It is of interest to note that locations with a continuous water supply actually exhibited a higher rate of infection. We speculate that this could be because the availability of running water leads to the existence of permanent bathtubs inside the homes, which may become sites for fungal growth. Further studies are required to verify this hypothesis. However, other non-environmental factors, such as a genetic predisposition to onychomycosis, may also play a role in this variation $[8,9]$.

Although $38.5 \%$ of confirmed cases were asymptomatic, clinical examination of the patients showed signs indicating the presence of a fungal infection. This observation suggests that an infection may develop without symptoms in the early stages in some anatomic areas, such as the interdigital region located of the foot. We recommend that clinicians carefully examine patients' feet for early detection and treatment of these initial lesions. Early detection will prevent further infection of other anatomical regions such as in nail beds [10]. The prevalence of onychomycosis in this study was higher $(3.37 \%)$ compared to the reported global prevalence in children $(0.3 \%)$ but similar to that reported in adults (3-8\%) [11].

Similar to the results of other studies, $T$. rubrum was the most common etiological agent of superficial fungal infections among teenagers in our setting. However, the occurrence of yeasts and Malassezia spp. was lower compared to the reports of other studies [12]. The presence of nonCandida albicans Candida species was remarkable as it was similar to levels reported in adults. In view of the saprophytic character of these aetiological agents and their recognized ability to develop resistance to the azoles, close clinical monitoring of these cases is recommended. These findings indicate that in our setting, the superficial fungal infections in teenagers have a different clinical behaviour compared to those seen in children in which scalp infection is more frequent. However, there is similarity with the clinical picture seen in adults, i.e., with more nail infections. T. rubrum, an anthropophillic fungus, is the predominant infection agent in teenagers.

In conclusion, asymptomatic superficial fungal infection caused predominantly by $T$. rubrum and Malassezia spp. occur in 38\% of teenagers in our setting. Occurrence of infection with Candida sp., which may promote development of resistance to azoles, is of concern. We recommend routine foot examinations for these patients by attending clinicians to promote early detection and treatment of these lesions.

\section{Acknowledgements}

Special thanks to the Superior Committee of Investigation of the Universidad Nacional Mayor de San Marcos for financing this investigation, my daughters Cristina, Denis and my friend Christine Feldmeier of Thomas Jefferson University School of Medicine.

\section{References}

1. Araj GF, Racoubian ES, Dather NK (2004) Etiologic agents of dermatophytes infection in Lebanon, J Med Liban 52: 59-63.

2. Fuentes Rivero D (2000) Epidemiologia y diagnostico clínico etiológico de onichomicosis en un centro medico universitario. Dermatology Peru 10: 21-33. 
3. Dolenc-Voljc M (2005) Dermatophyte infections in the Ljubljana region, Slovenia. 1995-2002. Mycoses 48: 181-186.

4. Truong A, Friedlander SF (2001) Superficial fungal infections in adolescence. Adolesc Med 12: 213-27.

5. Prohic A, Ozegovic L (2007) Malassezia species isolated from lesional and non-lesional skin in patients with pityriasis versicolor. Mycoses; 50: 58-63.

6. Bonifaz A (2000) Onychomycosis in Children: Treatment with Bifonazole-Urea. Pediatric Dermatology 17: 310-314.

7. Welsh O, Welsh E, Ocampo-Candiani J, Gomez M, Vera-Cabrera L (2006) Dermatophytoses in Monterrey, México. Mycoses_49: 119-123.

8. Lange M, Nowicki R, Baran W (2004) Dermatophytosis in children and adolescents in Gdansk, Poland. Mycoses 47: 326-329.

9. Ogasawara Y, Hiruma M, Muto M, (2003) Clinical and mycological study of occult tinea pedis and tinea unguium in dermatological patients from Tokyo. Mycoses 46: 114-119.

10. Cheng S, Chong L (2002) A prospective epidemiological study on tinea pedis and onychomycosis in Hong Kong. 115: 860-865.
11. AK Gupta (2004) Onychomycosis in Children: A brief overview with Treatment strategies. Pediatric Dermatology 21: 74-79.

12. Acosta Quintero ME, Cazorla Perfetti DJ (2004) Clinical-epidemiological aspects of pytiriasis versicolor (PV) in a fishing community of semiarid region from Falcon State, Venezuela. Rev Iberoam Micol 21: 191194.

\section{Corresponding Author \\ Juan Medina Flores \\ Children's Health National Institute \\ Department of Pediatrics \\ Brasil Av. Nº00 Breña \\ Lima Peru \\ Tel: 00-51-1-330-0066; 00-51-1-460-9875 \\ Email: jpmfha@yahoo.com}

Conflict of interest: No conflict of interest is declared. 